\title{
Universität Bielefeld
}

\section{Generating Useful Test Data for Complex Linked Employer-Employee Datasets}

\author{
Peter Jacobebbinghaus
}

German Data Service Center for Business and Organizational Data (DSZ-BO), Bielefeld

Research Data Centre of the Institute for Employment Research (FDZ-IAB), Nuremberg

IASSIST - May 30, 2013 


\section{Access to sensitive micro data}

- Data Anonymization is not feasible for all kinds of micro data (e.g. firm data)

- anonymization methods are hard to implement

- severe loss of information/analysis potential

- Access is provided

1 on-site in "save harbors"

2 remote execution of statistical programs

3 remote access 


\section{Access to sensitive micro data}

- Access modes 1 and 2 are costly

- time spent at RDCs

- number of jobs sent to RDCs

- Costs can be reduced by good preparation of program codes

- ahead of on-site use

- ahead of remote job submissions

- preferably in the researcher's office 


\section{Goals of test data}

- Test data (dummy data, structural data files,...)

- allow to write code

- allow to check program syntax

- any place: in researcher's office, on the train (anonymized)

- easy to create 


\section{Universität Bielefeld}

\section{Test data vs. usual anonymization}

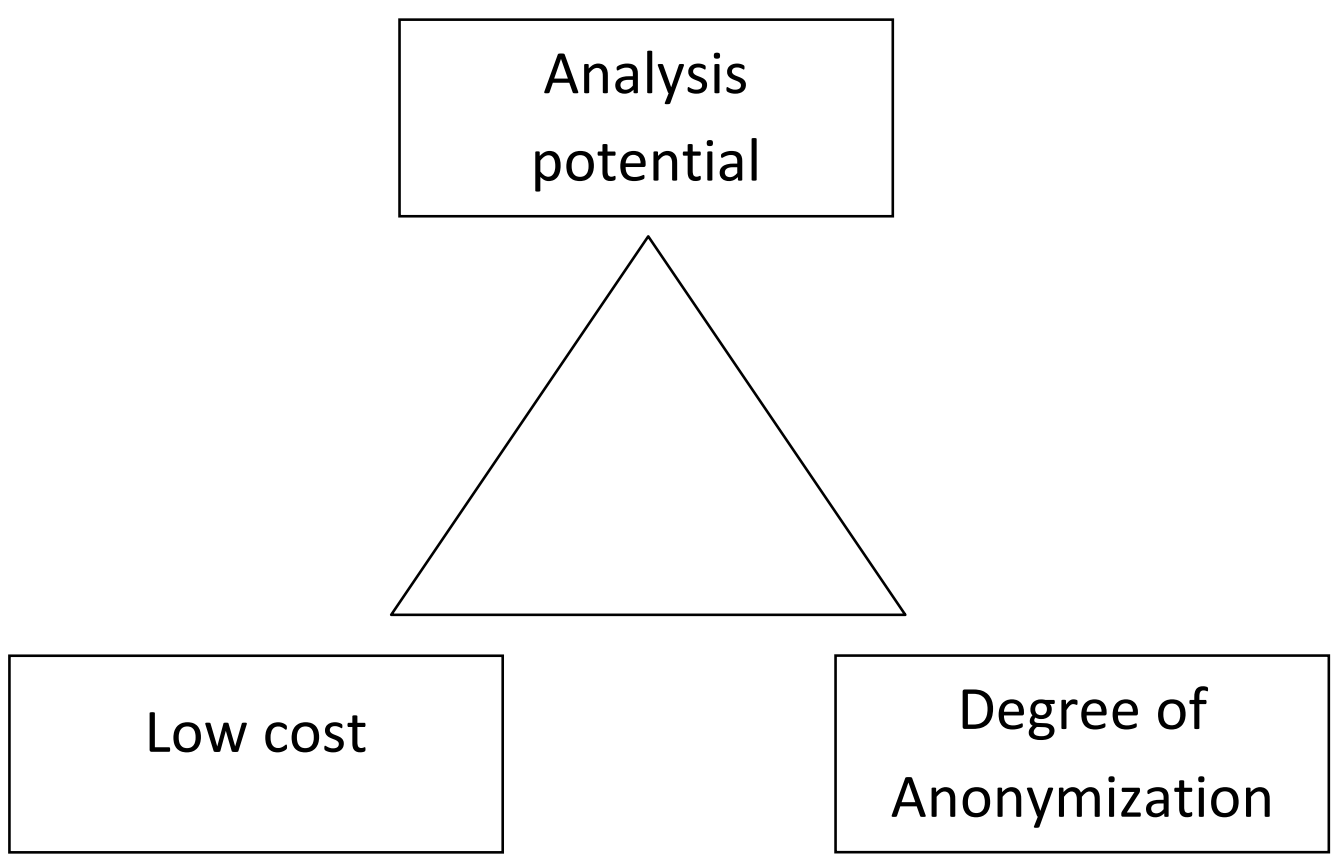




\section{Universität Bielefeld}

\section{Test data vs. usual anonymization}

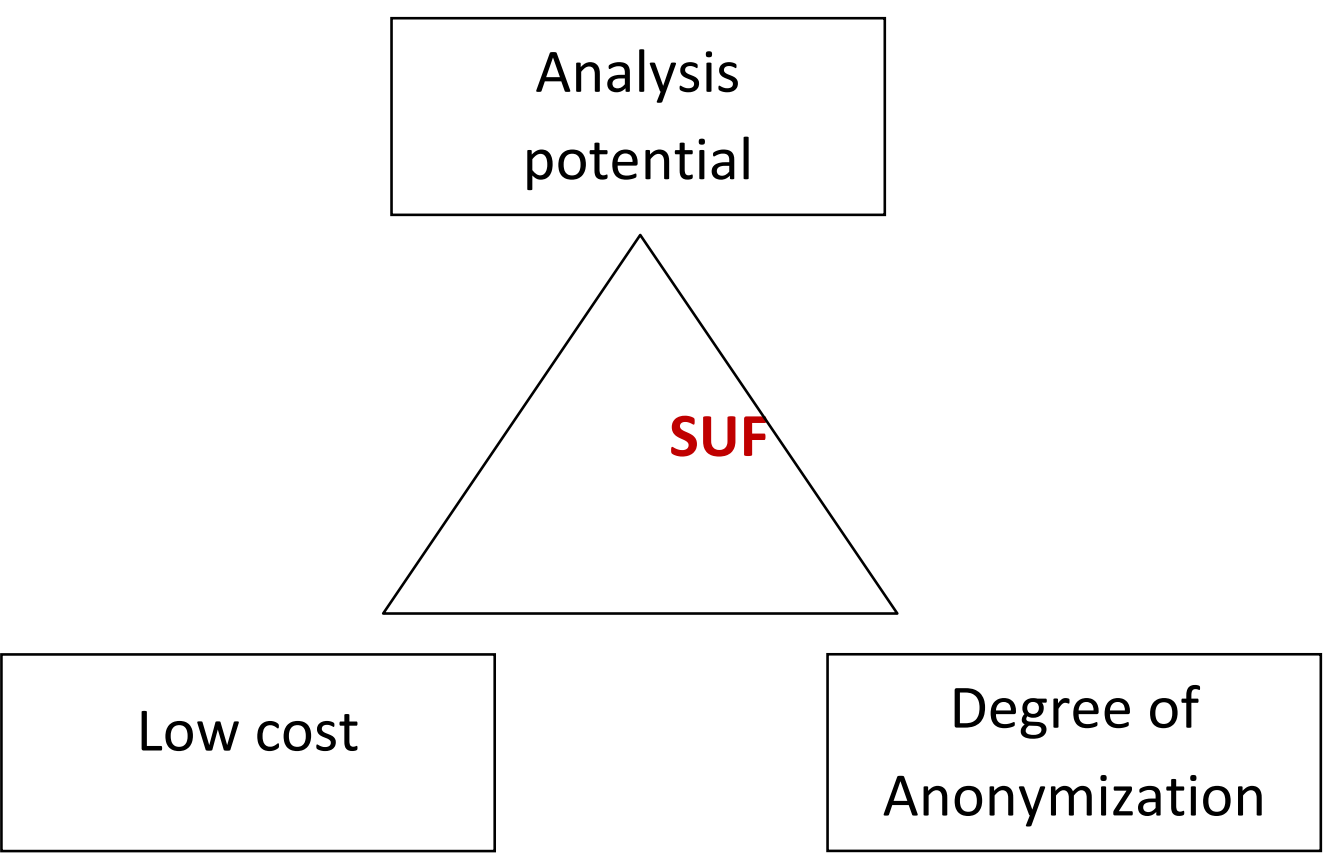




\section{Universität Bielefeld}

\section{Test data vs. usual anonymization}

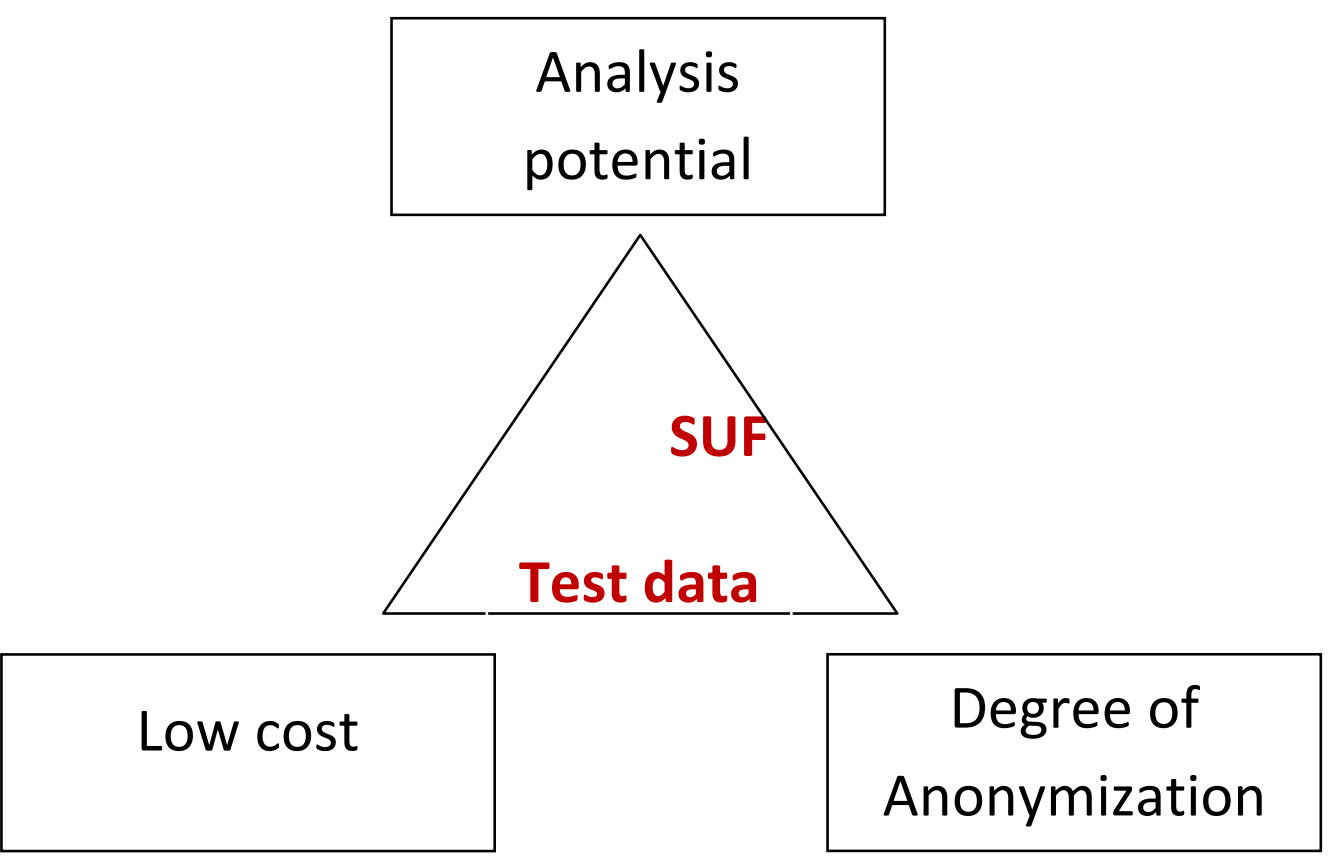




\section{Requirements of useful test data}

- Include all variables found the original data

- All values

- Keep key elements of the data structure

- panel data: entries, exits

- multilevel:

o Households, parents, children

o Regions, residents, movers

o LEE-data: employers and employees 


\section{How to do it}

- Restricted value swapping

- Complemented by

- random sample

- top/bottom coding or rounding

- check of string variables 


\section{Restricted value swapping}

Original data

\begin{tabular}{|c|c|c|}
\hline ID & size & region \\
\hline 1 & 23 & east \\
\hline 2 & 2 & east \\
\hline 3 & 13 & west \\
\hline 4 & 400 & west \\
\hline 5 & 11 & west \\
\hline
\end{tabular}

Swapped data

\begin{tabular}{|c|c|c|}
\hline ID & size & region \\
\hline 1 & 13 & west \\
\hline 2 & 2 & east \\
\hline 3 & 400 & west \\
\hline 4 & 11 & east \\
\hline 5 & 23 & west \\
\hline
\end{tabular}




\section{Universität Bielefeld}

\section{Restricted value swapping}

- Most datasets can be seen as one single matrix (wide format)

- one subject per row

- variables (and years) in columns

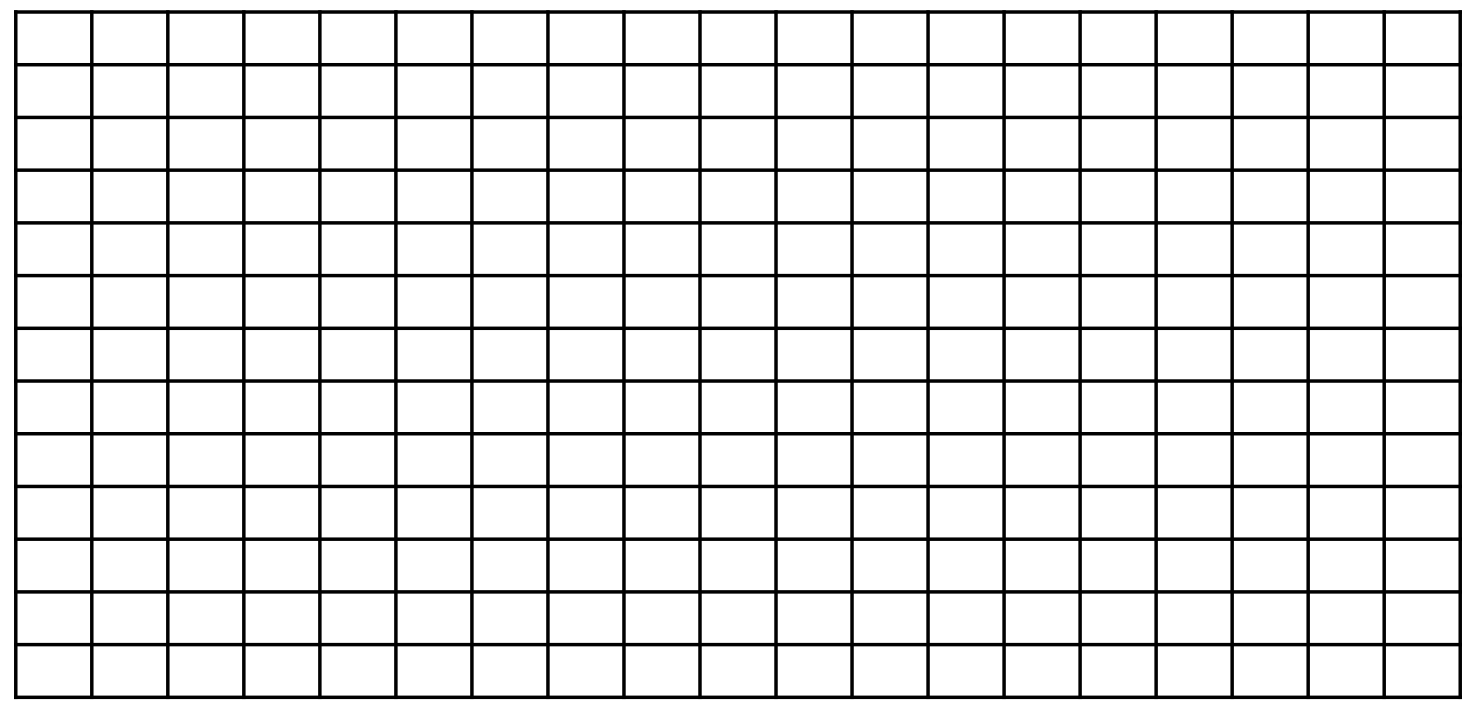




\section{Universität Bielefeld}

\section{Restricted value swapping}

- Most datasets can be seen as one single matrix (wide format)

- one subject per row

- variables (and years) in columns

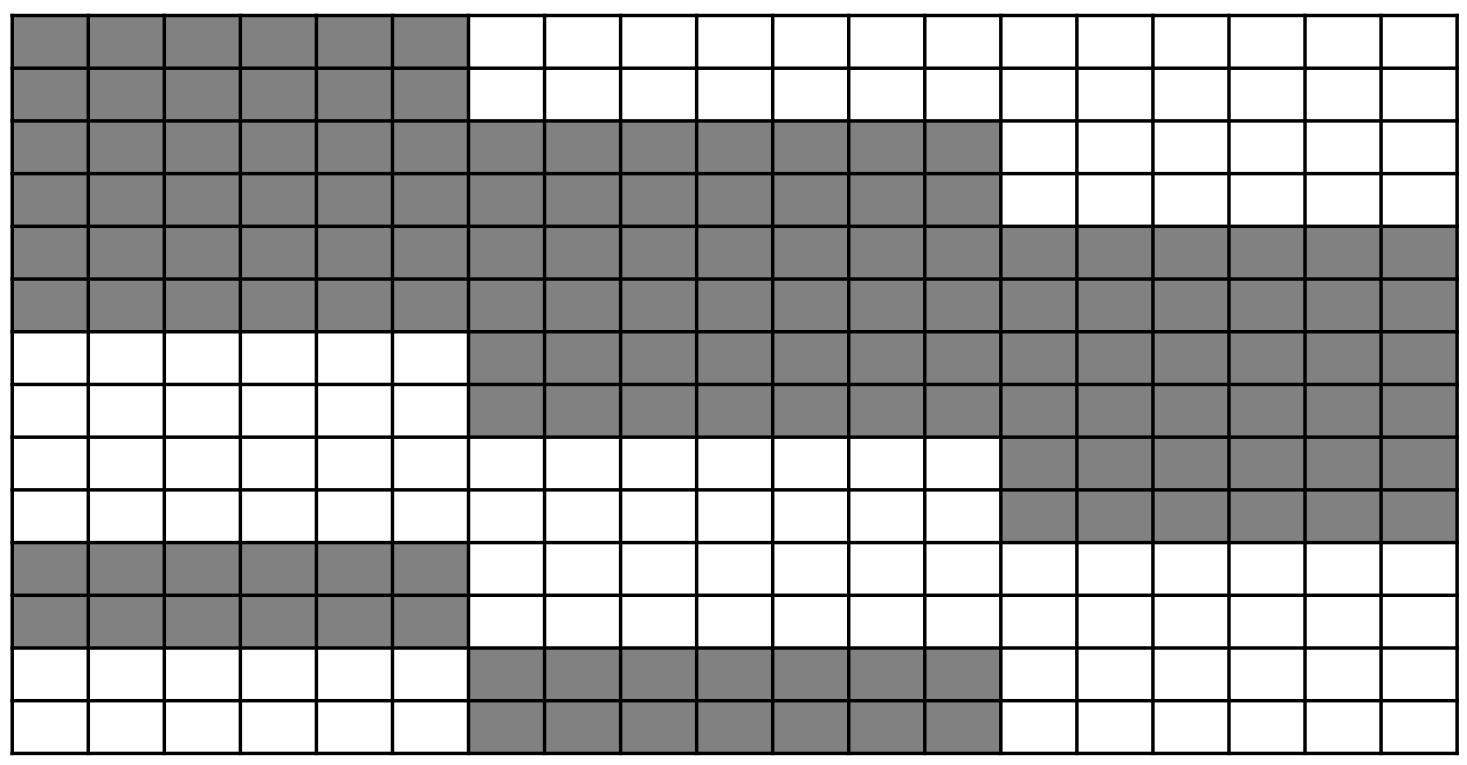




\section{Universität Bielefeld}

\section{Restricted value swapping}

- Swap values between subjects within the same pattern $\Rightarrow$ keep structure and some consistency (eg. over time)

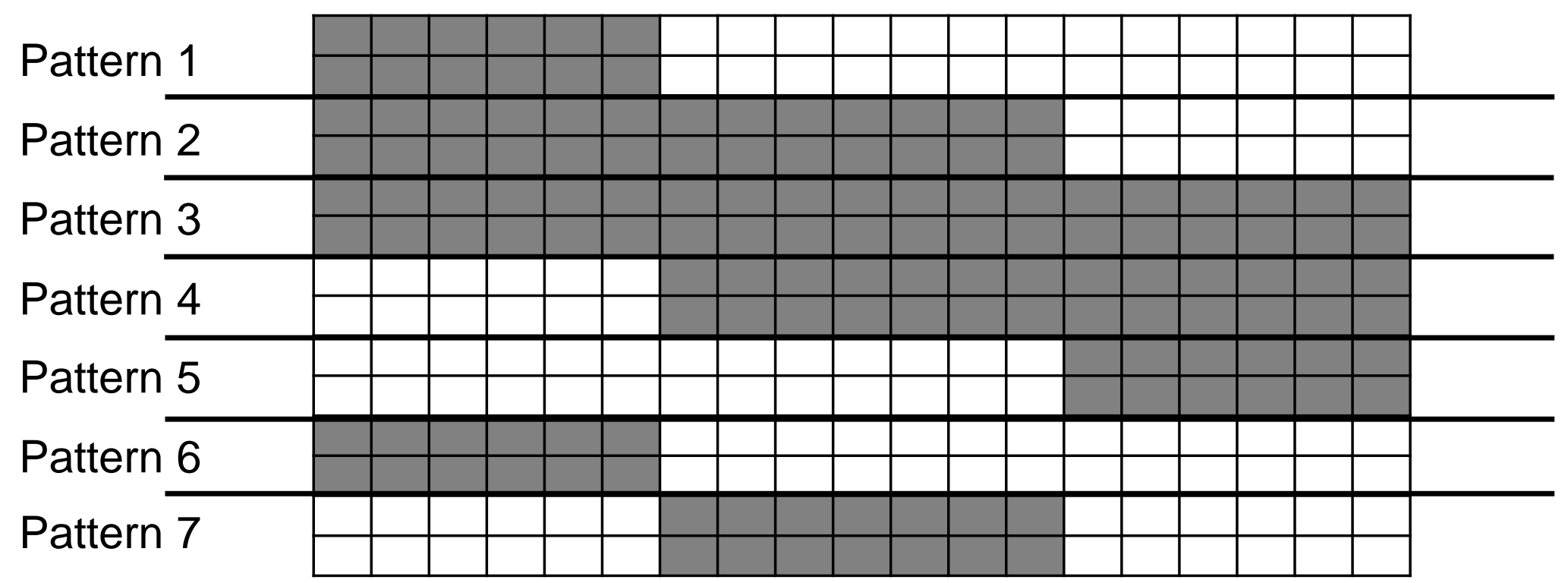




\section{Restricted value swapping}

- Swap values within subjects with the same pattern

=> keep structure and some consistency (eg. over time)

- if possible: also between similar observations

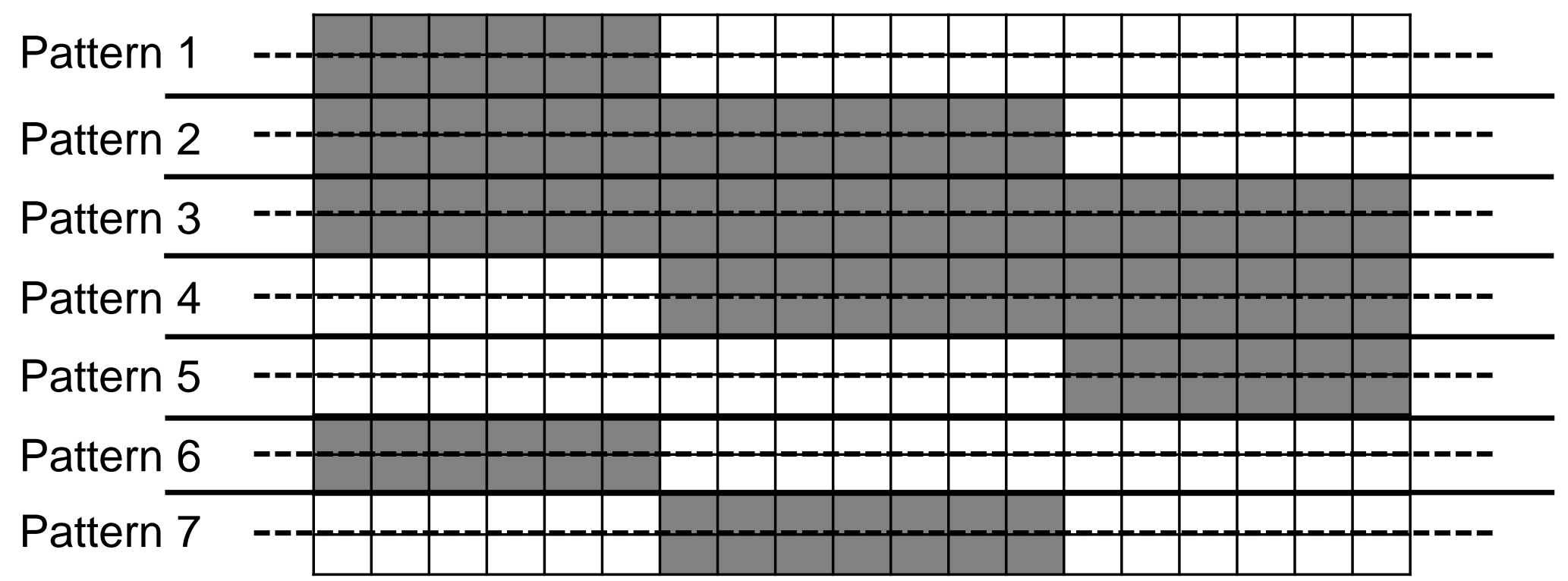




\section{Restricted value swapping}

- Keep additional structure by grouping variables

e.g. V1: variables on emloyee structure

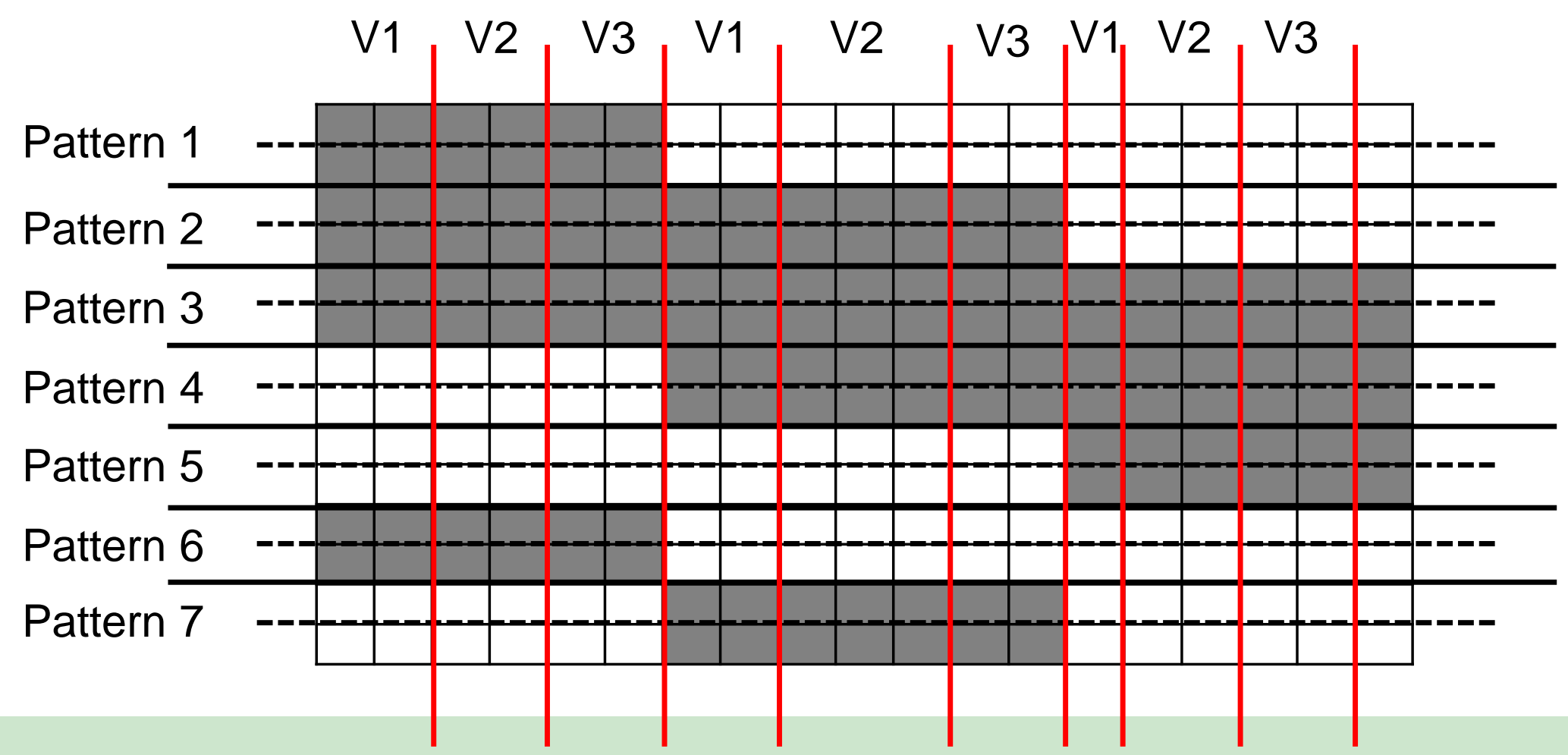




\section{Restricted value swapping}

- Keep additional structure by grouping variables

e.g. V1: variables on emloyee structure

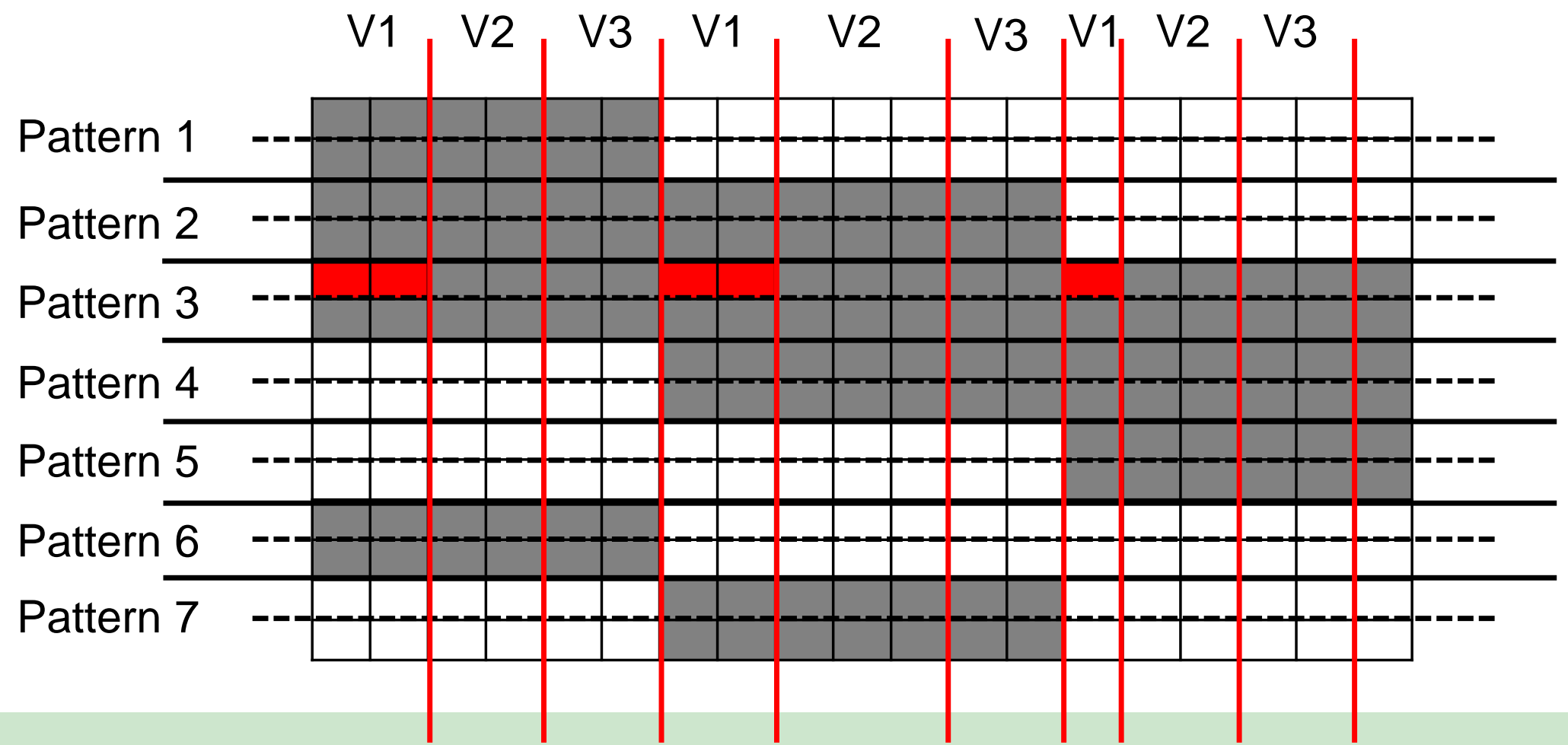




\section{Steps}

1 Identify patterns

2 Group similar observations within patterns

3 Drop all observations in small groups

4 Group related variables

5 Mark variables in need of additional measures (e.g. rounding)

6 Run swapping routines 


\section{Specifics of LEE-Data}

- How to keep the EE-matches?

- How to allow for movers?

1 Select sample of firms

2 Select all employees of these firms

3 Apply RVS to employer and employee data separately

4 Change the bipartite graph

o i.e.: who works for which firm in which year

$\mathrm{o}$ is altered by swapping firm ids among individuals with equal observation patterns 


\section{Implementations}

- IAB Establishment Panel: firm panel with 16 waves

- Sample of Integrated Labour Market Biographies (SIAB): register data on 2 mill. individuals, spell format

- Linked Employer-Employee Data from the IAB (LIAB)

- ...

- LEE ALLBUS 2008 Establishment Data (ALLBUS-BB)

- Employment Relationships and Social Exchange (BEATA)

- SFB580-B2 Establishment Panel 


\section{Pros and Cons}

- Advantages

- partially automated, easy to implement

- degree of anonymization can be adjusted

- tested procedure

- Limitations

- inconsistent variables: e.g. date of birth later than interview $\Rightarrow>$ if these are key variables manual corrections necessary

- selection of observations might result in loss of sparse values

- not every program checked with test data will work 


\section{Credits}

- Developed and applied

- Research Data Centre of the Institute for Employment Research (FDZ-IAB), Nuremberg (Dana Müller, Matthias Dorner)

- Data Service Center for Business and Organizational Data (DSZ-BO), Bielefeld

- Refined within BLUE-ETS project, 7th Framework EU project by Jörg Drechsler

=> programs (Stata) will be available at http://www.blue-ets.istat.it/ (deliverables WP 7.1) 